\title{
Contenidos Mínimos de Cariología e indicadores de aplicación clínica en el Currículo de Pregrado para las Escuelas Dentales Chilenas.
}

\section{Minimum Contents of Cariology and Indicators of Clinical Application in the Undergraduate Curriculum for Chilean Dental Schools.}

\begin{abstract}
Aránguiz V. ${ }^{1 *}$; Giacaman R.A. ${ }^{2}$; Cabello R. ${ }^{3}$., González S. ${ }^{4}$; Jans A. ${ }^{5}$; Mustakis A. ${ }^{6}$; Moncada G. ${ }^{1}$
1. Facultad de Odontología, Universidad de los Andes, Chile.

2. Unidad de Cariología, Departamento de Rehabilitación Buco-máxilofacial, Facultad de Ciencias de la Salud, Universidad de Talca, Talca, Chile.

3. Facultad de Odontología, Universidad de Chile, Santiago, Chile.

4. Facultad de Ciencias, Escuela de Odontología, Universidad Mayor, Chile

5. Facultad de Odontología, Universidad de La Frontera, Temuco, Chile.

6. Escuela de Odontología, Facultad de Medicina, Universidad Católica de Chile, Santiago, Chile.

* Correspondencia Autor: Vicente Aranguiz | Dirección: El Director 6000 Of. 209, Las Condes Santiago, Chile | Teléfono: +562 25017960 |

RESUMEN

Objetivo: El objetivo fue definir los contenidos mínimos y sus indicadores de aplicación clínica en el currículo de cariología para las escuelas de odontología chilenas. Metodología: Basados en los 5 dominios curriculares internacionales, se elaboró un documento que define los contenidos e indicadores de aplicación clínica para la enseñanza de cariología en Chile. Posteriormente, profesores de cariología de 20 de 21 escuelas de odontología chilenas $(95 \%)$, sesionaron para revisar, retroalimentar y elaborar el documento final, denominado "Listado de contenidos mínimos e indicadores de aplicación clínica" en cariología para estudiantes de pregrado de odontología en Chile. Resultados: Se definieron 23 contenidos y 31 indicadores de aplicación clínica para la enseñanza de la cariología. La cantidad de contenidos e indicadores separados por dominio fueron respectivamente: conocimiento de base: 5 y 7; riesgo/detección y diagnóstico: 6 y 6; toma de decisiones/manejo preventivo no operatorio: 5 y 5 ; decisión de tratamiento operatorio: 4 y 9 y cariología basada en la evidencia: 3 y 4 . Conclusiones: Se definieron los contenidos mínimos que tributan a cada dominio y sus indicadores de aplicación clínica para la enseñanza de la cariología en Chile.
\end{abstract} E-mail: vicente.aranguiz@gmail.com

Trabajo recibido el 08/09/2020

Trabajo revisado 21/03/2021

Aprobado para su publicación el 06/04/2021

PALABRAS CLAVE

Educación dental; Caries; Cariología; Contenidos; Indicadores clínicos; Currículo.
Int. J. Inter. Dent Vol. 14(2); 152-157, 2021.

\section{ABSTRACT}

The objective was to define the minimum contents and their indicators of clinical application in the cariology curriculum for the Chilean Dental Schools. Methodology: Based on the 5 international curricular domains, a document defining the contents and indicators of clinical application for the teaching of cariology in Chile was elaborated. Later, cariology professors from 20 out of the 21 Chilean Dentistry Schools (95\%) met to review, feedback and elaborate the final document, called "List of minimum contents and indicators of clinical application" in cariology for undergraduate dentistry students in Chile. Results: Twenty-three contents and 31 indicators of clinical application for the teaching of cariology were agreed upon. The amount of contents and indicators separated by domain respectively were: basic knowledge: 5 and 7; risk/detection and diagnosis: 6 and 6; decision making/non-operative preventive management: 5 and 5; decision of operative treatment: 4 and 9 and evidence-based cariology: 3 and 4. Conclusions: The minimum contents for each domain and its clinical application indicators for the teaching of cariology in Chile were defined.

\section{KEY WORDS}

Dental education; Caries; Cariology contents; Clinical indicators; Curriculum.

Int. J. Inter. Dent Vol. 14(2); 152-157, 2021.

\section{INTRODUCCIÓN}

La caries dental es la enfermedad crónica no transmisible más común del ser humano, el $35 \%$ de la población a nivel mundial presenta lesiones de caries no tratadas y en Latinoamérica esta cifra es cercana al $45 \%^{(1)}$. La caries afecta la calidad de vida y principalmente la autoestima, mediante el impacto en la función masticatoria y estética tanto en niños como en adultos ${ }^{(2-5)}$.

El enfoque basado en el manejo operatorio o quirúrgico de la caries dental aún prevalece entre los profesionales de la odontología, y los dientes ingresan al ciclo de re-restauración tal vez innecesariamente o demasiado pronto(6), lo que compromete el capital biológico de la persona, aspecto muy relevante en el contexto de un país que envejece aceleradamente y que en pocos años contará con cerca del $30 \%$ de su 
población adulta mayor.

Se ha demostrado que los dentistas pueden confiar en la creciente evidencia acumulada en cariología con respecto a las opciones de manejo menos invasivas de la enfermedad caries dental y se ha observado que cambios en la educación de los profesionales conducen a reducción de las intervenciones restauradoras ${ }^{(5)}$. Por otra parte, se ha observado que cuando se desarrolla un protocolo basado en la evidencia para el tratamiento no quirúrgico u operatorio de lesiones de caries y se monitorea esta práctica, es posible reducir el riesgo de experiencia de caries.

Existe consenso que los esfuerzos de la profesión por reducir la prevalencia de la enfermedad caries dental, requiere de cambios importantes en los planes y estrategias educativas en la enseñanza de pregrado, para lograr mayores avances en su prevención y control. En esa dirección, la educación puede ayudar a estrechar las brechas entre la investigación y la práctica clínica, para acelerar la adopción de estrategias basadas en la evidencia para el manejo de la enfermedad de caries y su consecuencia: las lesiones de caries $^{(5)}$. De hecho, se cuenta con gran cantidad de información recolectada acerca del currículo de cariología que se enseña en diversas escuelas de odontología del mundo ${ }^{(7-10)}$ y que han servido de fundamento para la construcción de currículos de consenso en Europa, Colombia, EE.UU. y Chile y que permite adaptaciones de acuerdo a las condiciones y reglamentación de cada país.

El cambio de paradigma de un modelo fundamentalmente restaurador a un modelo mixto y otro mínimamente invasivo se basa en la fuerza de la evidencia construida durante los últimos años que permiten reconocer que la enfermedad caries en etapas incipientes no requiere tratamiento quirúrgico del tejido dentario, determinando una filosofía que disminuye la frecuencia de las intervenciones quirúrgicas al ser cambiadas por acciones no invasivas, que junto a la reparación de restauraciones conservan el remanente biológico sano, disminuyen los tiempos de tratamiento y aumentan la vida útil de los dientes ${ }^{(24)}$

En el caso de Chile, se han efectuado tres estudios que establecen la situación de la educación de la cariología. El primero define el estado actual de la enseñanza de la cariología ${ }^{(11)}$, el segundo determina sus contenidos, metodología educativa y nivel de aplicación clínica actual ${ }^{(12)}$ y el tercero presenta un currículo por competencias construido con el consenso de la mayoría de las escuelas de odontología del país ${ }^{(13)}$.

Estudios previos en Chile sobre los contenidos y su aplicación en la clínica universitaria, detectaron que la mayoría de los contenidos encuestados se enseñaban y se impartían en cursos disciplinares teóricos o como contenidos integrados transversalmente en el currículo, sin embargo, se evidenciaba escasa aplicación en la práctica clínica universitaria ${ }^{(12)}$, similar a lo observado en Nueva Zelandia y Australia, en que a pesar que los modernos conceptos teóricos de cariología son enseñados, ellos no son necesariamente trasladados en la enseñanza clínica ${ }^{(14)}$. Adicionalmente, en Canadá se encontró que todas las escuelas carecen de la adecuada integración de cariología en el entrenamiento clínico ${ }^{(16)}$, confirmando el desbalance entre los tratamientos preventivos y los quirúrgicos en el manejo de las lesiones de caries dental.

Por otra parte, la inclusión de los indicadores clínicos, definidos como mediciones del proceso, estructura y resultados de la atención al paciente, son utilizados por los sistemas y servicios de salud, así como por los organismos de acreditación y regulación, para identificar áreas de preocupación que podrían requerir revisión o desarrollo adicional. Las características claves de un indicador ideal son: a) que se base en definiciones acordadas y se describe de manera exhaustiva y exclusiva; b) que sea altamente u óptimamente específico y sensible, es decir, que detecte pocos falsos positivos y falsos negativos; c) que sea válido y fiable; d) que discrimine adecuadamente; e) que se relacione con eventos claramente identificables para el usuario (por ejemplo, si está destinado a la formación de profesionales, es relevante para la práctica clínica); f) que permita comparaciones útiles; y g) que se base en la evidencia(15).

Los contenidos actuales de cariología, responden al cambio de paradigma de la enfermedad caries, según lo establecido por ORCA (The European Organization for Caries Research), la Unión Europea, EE.UU y Colombia y Chile ${ }^{(7-9,13)}$, en sus definiciones de competencias. La diferencia se observa principalmente en la metodología de trabajo, donde las escuelas dentales europeas iniciaron la construcción del currículo revisando los contenidos y luego el establecimiento de la estructura curricular por dominios y competencias, mientras que en Chile estas últimas se utilizaron como base para la definición de los contenidos.

Los progresos e implementación de cambios curriculares durante los últimos años han sido modestos para incorporar la evidencia del cambio de paradigma para el manejo de la enfermedad caries en la clínica y de las lesiones resultantes. La integración de currículos básicos y clínicos es un conflicto frecuente en las áreas de salud en el mundo, ya el año 1995 el instituto de medicina de EE.UU proponía realizar currículos integrados, especialmente si se observa la disminución de las horas de formación básica tanto en medicina como en odontología en las universidades norteamericanas durante las últimas décadas, dada la integración de currículos $^{(19-21)}$.

Dado que en Chile los contenidos no están consensuados entre las escuelas de odontología y que no existe un precedente de un proceso nacional para aunar criterios en esta materia, el objetivo del presente estudio fue definir los contenidos y sus indicadores de aplicación clínica en el currículo de cariología para las escuelas de odontología chilenas de pregrado. El artículo reporta el proceso utilizado, el análisis de contenidos de los currículos en otros países y sus posibles limitaciones.

\section{METODOLOGÍA}

\section{El proceso fue planificado en tres etapas:}

Etapa 1: El Comité de Cariología ( $\mathrm{CC}=$ organización académica creada por los profesores de cariología de las universidades chilenas) definió el listado de contenidos y su aplicación clínica en el período de seis meses, considerando como base los 5 dominios establecidos inicialmente por la Unión Europea (UE), y posteriormente adaptados por EE.UU. y Colombia $^{(8-10,13)}$, además de los consensuados en Chile, trabajo liderado por el CC durante 2017-2018(13), definidos como: 1 . Conocimientos base; 2. Determinación de riesgo, diagnóstico caries y detección de lesiones de caries; 3 . Toma de decisiones y manejo preventivo no operatorio/ no quirúrgica; 4. Toma de decisiones y terapia operatoria/quirúrgica y 5. Cariología basada en la evidencia, en la práctica clínica y en salud pública, enunciando los contenidos bajo títulos generalizadores, no incluyendo nombres propios y elaborando indicadores de aplicación clínica para cada uno de los contenidos, esto último, considerando que en la secuencia del aprendizaje y la competencia profesional se evalúan en su aplicación clínica.

Etapa 2: EI CC envió una carta de invitación a todos los decanos o directores de escuela, según correspondiera en cada institución de educación superior, para participar en la discusión y definición de los contenidos y sus correspondientes indicadores clínicos. Para ello, se solicitó que designaran al o a los representantes de cariología de sus respectivas unidades académicas. Este proceso de invitación y constitución de los representantes de las escuelas se extendió durante tres meses. Esta fase se inició con el envío y acuso de recibo de la propuesta, solicitándose a cada uno de los profesores (as), que con toda libertad agregaran, modificaran o eliminaran contenidos e indicadores como parte del proceso de retroalimentación.

El propósito de esta etapa fue construir un segundo documento para ser sometido a consenso y validación de la propuesta final, mediante una sesión presencial en el ámbito del IV Encuentro Internacional de Cariología de Chile.

Etapa 3: En esta etapa los contenidos y sus indicadores fueron sometidos al análisis, discusión y acuerdo entre profesores de Cariología de 20 de las 21 escuelas dentales chilenas (95\%).

Esta reunión presencial se desarrolló en dos fases destinadas al análisis pedagógico y filosófico de la enseñanza de la cariología de acuerdo a las necesidades del entorno y la problemática país. La primera fase incluyó dos presentaciones introductorias, sobre la importancia del currículo de consenso ${ }^{(13)}$ y sobre la necesidad de generar un listado de contenidos y sus indicadores de aplicación clínica.

En la segunda fase los asistentes se distribuyeron en tres grupos de trabajo, cada grupo analizó, discutió y consensuó los contenidos e indicadores clínicos asociados a los dominios correspondientes. A continuación, en la sesión plenaria, cada grupo presentó sus propuestas, las que fueron sometidas a la aprobación o rechazo por la totalidad de los asistentes, teniendo en consideración la experiencia, la valoración y sentido de pertinencia de los dominios en los futuros planes curriculares.

El documento final fue sometido a la corrección de aspectos formales de redacción por los miembros del comité y finalmente los contenidos e indicadores aprobados por unanimidad fueron entregados a ACHEO (Asociación Chilena de Enseñanza de la Odontología).

\section{RESULTADOS}

Con la participación del $95 \%$ de las facultades y escuelas de odontología de las universidades chilenas fueron definidos y aprobados por unanimidad el listado de contenidos mínimos e indicadores de aplicación clínica para la enseñanza de la cariología, en concordancia con los dominios del currículo mínimo de cariología, previamente desarrollados en el país y publicado el año $2018^{(13)}$.

Se acordaron en total 23 contenidos mínimos y 31 indicadores de 
aplicación clínica para la enseñanza de la cariología en Chile. La cantidad de contenidos e indicadores separados por dominio, respectivamente fueron: Dominio 1: Conocimiento de base: 5 y 7; Dominio 2: Riesgo; detección y diagnóstico: 6 y 6 . Dominio 3: Toma de decisiones y manejo preventivo no operatorio: 5 y 5 . Dominio 4: Decisión de tratamiento operatorio: 4 y 9, y Dominio 5: Cariología basada en la evidencia: 3 y 4 (Tabla 1)

Tabla 1: Listado de contenidos mínimos e indicadores para la enseñanza de la cariología en las escuelas de odontología chilenas, por Dominio del Currículo de Competencias en Cariología.

\begin{tabular}{c|l}
\hline DOMINIO 1 & \multicolumn{1}{c}{ Contenidos } \\
\hline & $\begin{array}{l}\text { C1 Anatomía y desarrollo de los tejidos dentarios y estruc- } \\
\text { turas de soporte a nivel macroscópico, microscópico y mo- } \\
\text { lecular/iónico }\end{array}$ \\
\cline { 2 - 2 }
\end{tabular}

C2 Concepto de enfermedad de caries, lesiones de caries y lesiones no cariosas. Rol del biofilm dental, dieta, saliva y progresión de la enfermedad

C3 Bases químicas, físicas y biológicas de los cambios en los tejidos duros, relacionados con la detección clínica y radiológica. Evaluación de lesiones de caries y otras lesiones no cariosas

C4 Principios básicos de profesionalismo: ética, bioética.

C5 Teorías sicológicas y técnicas para promover el cambio de comportamientos y hábitos para mejorar la salud bucal. Herramientas básicas para la comunicación efectiva.

\section{Indicadores de aplicación clínica}

I.1 Reconocimiento del desarrollo y morfología de la anatomía dentaria.

I.2 Identificar y utilizar protocolos de evaluación de riesgo o factores asociados al riesgo individual del paciente.

I.3 Detectar lesiones cariosas y no cariosas a nivel clínico y radiográfico utilizando un criterio validado.

I.4 Realizar valoración de la actividad de las lesiones de caries a nivel clínico.

I.5 Otorgar atención odontológica respetando los principios bioéticos.

I.6 Detectar necesidades de modificación de cambios de comportamiento y hábitos para mejorar la salud oral.

I.7 Realizar la derivación oportuna al profesional de la salud que corresponda.

\begin{tabular}{c|c|c}
\hline DOMINIO 2 & \multicolumn{1}{|c|}{ Contenidos } & \multicolumn{1}{c}{ Indicadores de aplicación clínica } \\
\hline & C6 Diagnóstico centrado en el paciente & $\begin{array}{l}\text { I.8 Evaluar e integrar antecedentes demográficos, socio } \\
\text { culturales, conductuales, sistémicos, clínicos, radiográficos } \\
\text { para emitir el juicio diagnóstico. }\end{array}$ \\
\cline { 2 - 3 }
\end{tabular}

C7 Evaluación de la enfermedad caries dental en el paciente considerando enfoque de curso de vida

C8 Evaluación de la salud sistémica del paciente y los efectos bucales derivados de su condición y/ o de sus tratamien-

Riesgo, detección y diagnóstico tos

C9 Criterios de detección clínico, severidad, actividad y efectos pulpares de las lesiones cariosas ries

C11 Diagnóstico diferencial de lesiones de caries
C10 Detección y evaluación radiográfica de lesiones de ca-

I.9 Identificar y aplicar indicadores, factores de riesgo y factores de pronósticos a nivel individual determinado su contexto social y comunitario, aplicados en el tiempo

I.10 Relacionar las enfermedades sistémicas y sus tratamientos con la enfermedad de caries dental

I.11 Aplicar sistemas estandarizados de detección y evaluación clínica de lesiones cariosas y del estado de salud pulpar

I.12 Aplicar criterios para la determinación de la severidad radiográfica de lesiones de caries

I.13 Aplicar criterios para la detección y diagnostico diferencial de defectos del esmalte y dentina; hipoplasias, amelogénesis imperfecta, crack dentario, fluorosis, $\mathrm{MIH}$, lesiones no cariosas (erosión, abrasión, abfracción, atrición), malformaciones dentarias y evaluar su origen).

\begin{tabular}{|c|c|c|}
\hline DOMINIO 3 & Contenidos & Indicadores de aplicación clínica \\
\hline \multirow{5}{*}{$\begin{array}{l}\text { Toma de decisiones y } \\
\text { manejo preventivo no } \\
\text { operatorio }\end{array}$} & C12 Cambios conductuales para promover la salud bucal & $\begin{array}{l}\text { I.14 Aplicar instrumentos de evaluación asociado a cambios } \\
\text { de conducta. }\end{array}$ \\
\hline & $\begin{array}{l}\text { C13 Asesoramiento y monitoreo dietético. Efectos de los } \\
\text { desórdenes alimentarios en la salud bucal. }\end{array}$ & $\begin{array}{l}\text { I.15 Identificar y utilizar instrumentos para evaluar y moni- } \\
\text { torear dieta }\end{array}$ \\
\hline & C14 Control mecánico, químico y biológico del biofilm dental & $\begin{array}{l}\text { I.16 Realizar instrucción de higiene oral, monitoreo de indi- } \\
\text { cadores y definición de intervalos de mantención. Remoción } \\
\text { profesional del biofilm. }\end{array}$ \\
\hline & $\begin{array}{l}\text { C15 Fluoruros: mecanismo de acción, presentaciones y uso } \\
\text { racional según nivel de riesgo y edad. }\end{array}$ & $\begin{array}{l}\text { I. } 17 \text { Indicar correctamente, prescribir y aplicar clínicamente } \\
\text { fluoruros, según nivel de riesgo y edad }\end{array}$ \\
\hline & C16 Otros agentes anticaries y remineralizantes. & $\begin{array}{l}\text { I.18 Indicar otros agentes distintos del fluoruro según nivel } \\
\text { de riesgo y edad }\end{array}$ \\
\hline
\end{tabular}


Tabla 1: Listado de contenidos mínimos e indicadores para la enseñanza de la cariología en las escuelas de odontología chilenas, por Dominio del Currículo de Competencias en Cariología. (continuación)

\begin{tabular}{|c|c|c|}
\hline DOMINIO 4 & Contenidos & Indicadores de aplicación clínica \\
\hline \multirow{9}{*}{$\begin{array}{l}\text { Decisiones de trata- } \\
\text { miento operatorio }\end{array}$} & \multirow{2}{*}{$\begin{array}{l}\text { C17. Fundamentos del tratamiento mínimamente invasivo } \\
\text { de lesiones de caries. }\end{array}$} & $\begin{array}{l}\text { I.19 Efectuar tratamiento micro y mínimamente invasivo en } \\
\text { lesiones cavitadas y activas. }\end{array}$ \\
\hline & & $\begin{array}{l}\text { 1.20 Priorizar reparación y sellado de restauraciones defec- } \\
\text { tuosas por sobre el remplazo total de la restauración. }\end{array}$ \\
\hline & \multirow{2}{*}{$\begin{array}{l}\text { C18. Diseño de preparaciones biológicas para tratamiento } \\
\text { de lesión de caries determinadas por actividad, ubicación, } \\
\text { extensión y profundidad. }\end{array}$} & $\begin{array}{l}\text { I.21 Realizar mínimo y suficiente acceso a la lesión, utilizan- } \\
\text { do instrumental apropiado. }\end{array}$ \\
\hline & & I.22 Remover selectivamente el tejido cariado. \\
\hline & \multirow{2}{*}{$\begin{array}{l}\text { C19. Manejo quirúrgico de las lesiones de caries que garan- } \\
\text { tice la mantención de la salud bucal. }\end{array}$} & I.23 Realizar acciones que protejan la pulpo dentinaria. \\
\hline & & $\begin{array}{l}\text { I.24 Efectuar reconstrucción morfológica de contactos pro- } \\
\text { ximales, contornos dentarios, textura superficial y contactos } \\
\text { oclusales funcionales }\end{array}$ \\
\hline & \multirow{3}{*}{$\begin{array}{l}\text { C20. Implementación de programas de control y manten- } \\
\text { ción, según evaluación de riesgo y pronóstico }\end{array}$} & $\begin{array}{l}\text { I.25 Determinar riesgo y pronóstico a nivel individual y/o co- } \\
\text { munitario. }\end{array}$ \\
\hline & & $\begin{array}{l}\text { I.26 Establecer frecuencia de control y mantención según } \\
\text { nivel de riesgo }\end{array}$ \\
\hline & & $\begin{array}{l}\text { I.27 Aplicar programa de control en pacientes de mantención } \\
\text { y monitorear su seguimiento }\end{array}$ \\
\hline DOMINIO 5 & Contenidos & Indicadores de aplicación clínica \\
\hline \multirow{4}{*}{$\begin{array}{l}\text { Cariología basada } \\
\text { en la evidencia en la } \\
\text { práctica clínica y de } \\
\text { Salud Pública }\end{array}$} & $\begin{array}{l}\text { C21. Desarrollo de programas de promoción, prevención y } \\
\text { mantención en salud oral. }\end{array}$ & $\begin{array}{l}\text { I.28 Conocer, diseñar y aplicar programas de promoción, } \\
\text { prevención y mantención de la salud oral. }\end{array}$ \\
\hline & $\begin{array}{l}\text { C22. Epidemiología de la caries en relación con las deter- } \\
\text { minantes de la salud }\end{array}$ & $\begin{array}{l}\text { I.29 Utilizar las herramientas cualitativas y cuantitativas de } \\
\text { la epidemiología. }\end{array}$ \\
\hline & \multirow{2}{*}{$\begin{array}{l}\text { C23. Fundamentar el quehacer clínico en la evidencia cien- } \\
\text { tífica disponible. }\end{array}$} & $\begin{array}{l}\text { I.30 Aplicar los conceptos de odontología basada en la evi- } \\
\text { dencia científica disponible. }\end{array}$ \\
\hline & & $\begin{array}{l}\text { I.31 Aplicar guías clínicas validadas para el manejo de la ca- } \\
\text { ries dental. }\end{array}$ \\
\hline
\end{tabular}




\section{DISCUSIÓN}

El listado de contenidos definidos en Chile, se alinea con lo establecido por ORCA, la Unión Europea, EE.UU, Colombia ${ }^{(7-9)}$, en sus definiciones de competencias. Un aspecto diferente, fue la no inclusión en Chile del contenido en relación a la caries temprana de la infancia ${ }^{(7)}$, hecho que podría explicarse porque este contenido es parte del programa del área de Odontopediatría. La bibliografía muestra que la cantidad de contenidos es variable, mientras la comunidad europea definió 27 , Brasil 29(17) y Chile 23, mostrando mínimas diferencias en los tópicos abordados ${ }^{(7,9)}$.

Durante el debate de la propuesta no se produjo el descarte de ningún contenido, sin embargo, se procedió a fusionar algunos, dado que abordaban conocimientos, habilidades y comportamientos comunes. En general, los fundamentos para la inclusión de los contenidos, destacaron por la asociación de cada uno de ellos con sus respectivos conocimientos, con la experiencia clínica, el pensamiento crítico y la habilidad para la resolución de problemas, junto a profesionalismo, valores éticos, habilidades técnicas y procedimentales ${ }^{(10)}$.

Los aspectos de mayor discusión fueron los contenidos asociados a la promoción de cambios conductuales en los pacientes, donde las herramientas básicas para la comunicación efectiva (D1 C5) y el manejo de los cambios conductuales para promover la salud bucal (D3 C1) representan un desafío para la formación de las futuras generaciones. El plenario reflexionó sobre la falta de competencias actuales para el manejo de estas variables conductuales, planteándose la posible incorporación de profesionales especialistas que faciliten la implementación de estos contenidos, en un marco de trabajo de las estrategias de aprendizaje que desarrollen habilidades específicas de esta área en los futuros profesionales $^{(18)}$

Existe un gran cambio entre los actuales contenidos enseñados y el listado consensuado, lo que motivará posiblemente a todos los académicos a reflexionar sobre la diversificación de los contenidos enseñados y reenfocar la actividad docente, con especial énfasis en dialogar con las asignaturas clínicas y favorecer el trabajo interdisciplinario, en busca de acoger el presente listado de contenidos.

Según los comentarios de los profesores de cariología participantes en la reunión, a dos años de la presentación del nuevo currículo de cariología en Chile, la gran mayoría de las escuelas no ha concretado procesos de implementación que conduzcan a la adopción de cambios curriculares. Esta propuesta de contenidos e indicadores presenta como principal ventaja su mayor flexibilidad y gradualidad de inserción de los contenidos en la formación transversal del pregrado, dado que es posible su inclusión en diferentes modelos educativos, tanto en aquellos planes de formación basados en competencias, como en los sustentados en resultados de aprendizaje o por objetivos. De hecho, los contenidos mínimos consensuados no definen que su entrega sea exclusivamente a través de una asignatura, ya que pueden ser distribuidos a lo largo de la malla curricular como contenido de varias asignaturas o módulos independientes, sin necesidad de que su entrega sea exclusivamente de uno o varios cursos formales de cariología. Estos contenidos pueden insertarse en los tres ciclos del aprendizaje: básico, preclínico y clínico y sus indicadores permiten evaluar la transferencia de los contenidos teóricos a la práctica clínica ${ }^{(9)}$.

Para el desarrollo de competencias profesionales sólidas se requiere específicamente de avances en los contenidos, aplicar pedagogías más eficaces y evaluar la preparación de los estudiantes ${ }^{(22)}$. La experiencia pedagógica publicada, señala que cada indicador de aplicación clínica debe ser determinado en detalle, con descripciones explícitas, sin embargo, la actual propuesta es de carácter general y voluntaria, entregando la responsabilidad para incrementar la especificidad y sensibilidad de cada indicador de acuerdo con la realidad educativa de cada universidad ${ }^{(23)}$

La información sobre contenidos de los currículos será relativamente fácil de recolectar a futuro, ya que una forma simple para realizar un primer análisis de la situación actual de cada facultad o escuela sería comparar los contenidos declarados en los programas de cursos con estos contenidos mínimos y así, auto evaluar el nivel de cumplimiento o conformidad con el currículo de cariología de consenso o para realizar el análisis de las posteriores adecuaciones necesarias al currículo actual.

No obstante, es preciso conocer datos sobre la efectividad de los procesos educativos y su impacto en los estudiantes, y en especial en los resultados de la atención al paciente, con el propósito de comprobar su real aplicación en las escuelas. Lo anterior constituye uno de los mayores desafíos a resolver en los próximos años, donde la principal dificultad que presentará este listado de contenidos será la evaluación en el tiempo de los indicadores de aplicación clínica y su efecto sobre la formación del odontólogo. Debe destacarse que en el país aún no existe una prueba de acreditación profesional, siendo la próxima tarea generar un instrumento para determinar el logro de los indicadores de aplicación clínica, o bien incorporar estos contenidos en una potencial prueba de habilitación profesional que surja en el país.

Durante los últimos años se ha determinado que el tema de la calidad de la educación superior, es uno de los principales desafíos de la educación terciaria chilena, junto con el rol del docente universitario, los contenidos educativos y sus efectos sobre los estudiantes, por esta razón la construcción de los contenidos e indicadores de aplicación clínica de Cariología en el currículo de Pregrado para las Escuelas Dentales Chilenas representa un importante aporte para educación de calidad en Odontología ${ }^{(25)}$.

\section{CONCLUSIONES}

Luego de una definición inicial, el Comité de Cariología invitó a participar a todas las escuelas de odontología del país para construir el listado de contenidos mínimos y sus Indicadores de aplicación clínica para la enseñanza de la cariología en los programas de pregrado. Estos se discutieron y aprobaron en metodología de trabajo grupal, con acuerdo unánime de cada uno de los contenidos mínimos e indicadores de aplicación clínica, que tributan a cada dominio para la enseñanza de la cariología en el pregrado.

\section{AGRADECIMIENTOS}

Los autores desean agradecer a los profesores de Cariología de las Facultades o Escuelas de Odontología de Chile designados para la definición de los Contenidos Mínimos en Cariología: Darwin D. Pérez Miranda, Ernesto González Mallea, Romina Andrea Bopp, Yuliana Candiales, María Eugenia Ortiz, Juan Eduardo Onetto, Alfredo Silva, Iván Urzúa, Vivi Muente, Begoña Ruiz, Ana M. Carrizo, Enrique Fernandez, Felipe Tagle, Gonzalo Rodríguez, Constanza Fernandez, Juliana Botelho, Vladimir Pizarro.

Asimismo, los autores desean brindar un reconocimiento a las autoridades de cada casa de estudios, decanos de facultad o directores de escuela, por la buena disposición a colaborar con este proceso.

\section{CONFLICTOS DE INTERÉS}

Los autores declaran no presentar conflictos de interés.

\section{FONDOS}

Ninguno 


\section{Bibliografía}

1. Kassebaum NJ, Bernabé E, Dahiya M, Bhandari B, Murray CJ and Marcenes W. Global burden of untreated caries: a systematic review and metaregression. J Dent Res. 2015;94:650-8.

2. Martins-Júnior PA, Vieira-Andrade RG, Corrêa-Faria P, Oliveira-Ferreira F, Marques LS and Ramos-Jorge ML. Impact of early childhood caries on the oral health-related quality of life of preschool children and their parents. Caries Res. 2013;47(3):211-8.

3. León S, Bravo-Cavicchioli D, Correa-Beltrán G and Giacaman RA. Validation of the Spanish version of the Oral Health Impact Profile (OHIP-14Sp) in elderly Chileans. BMC Oral Health. 2014;4;14:95.

4. Haag DG, Peres KG, Brennan DS. Tooth loss and general quality of life in dentate adults from Southern Brazil. Qual Life Res. 2017;26(10):2647-57.

5. Fontana M, Zero D. Bridging the gap in caries management between research and practice through education: the Indiana University experience. J Dent Educ. 2007;71(5):579-91.

6. Elderton RJ. Preventive (evidence-based) approach to quality general dental care. Med Princ Pr. 2003;12 Suppl 1:12-21.

7. Schulte AG, Buchalla W, Huysmans MC, Amaechi BT, Sampaio F, Vougiouklakis $G$, et al. A survey on education in cariology for undergraduate dental students in Europe. Eur J Dent Educ. 2011;15(1):3-8.

8. Martignon S, Gomez J, Tellez M, Ruiz JA, Marin LM, Rangel MC. Current cariology education in dental schools in Spanish-speaking Latin American countries. J Dent Educ. 2013;77(12):1330-7.

9. Ferreira-Nóbilo NP, de Sousa MLR,Cury JA. Cariology in curriculum of Brazilian dental schools. Braz Dent J. 2014;25(4):265-70.

10. Fontana M, Guzmán-Armstrong S, Schenkel AB, Allen KL, Featherstone J, Goolsby S, et al. Development of a core curriculum framework in cariology for U.S.dental schools. J Dent Educ. 2016;80(6):705-20.

11. Diaz-Yokens M, González S, Giacaman R, Araya-Bustos M, Moncada G, Martignon S. Cariology curriculum in Chilean universities. Rev Clin Periodoncia Implant Rehabil Oral. 2018;11(2):98-101.

12. Aránguiz V, Marró ML, Ramírez V, Moncada G. Contents of cariology taught by Chilean dental schools: Cross-sectional study. Rev Clin Periodoncia Implant Rehabil
Oral. 2019;12(1):31-6.

13. Giacaman R, Aranguiz V, Botelho J, Bustos I, Cabello R, et al. Development of a competency-based curriculum in cariology for undergraduate dental students in Chile. Rev Clin Periodoncia Implant Rehabil Oral. 2018;11(1);58-68.

14. Raphael SL, Foster Page LA, Hopcraft MS, Dennison PJ, Widmer RP, Evans RW. A survey of cariology teaching in Australia and New Zealand. BMC Med Educ. 2018 Apr 10;18(1):75

15. Mainz J. Defining and classifying clinical indicators for quality improvement, International. Int J Qual Health Care. 2003;15(6):523-30.

16. Tikhonova S, Girard F, Fontana M. Cariology education in Canadian dental schools: where are we? where do we need to go? J Dent Educ. 2018;81(1):39-46. 17. Gouvea D, Groisman S, Strazeri MJ, Sampaio F, Paiva SM, Kriger L et al. Cariology education for undergraduate Brazilian dental students. Rev Gaúch Odontol. 2018:66(3):239-44.

18. Nicolaides M, Cardillo L, Theodoulou I, Hanrahan J, Tsoulfas G, Athanasiou T, et al. Developing a novel framework for non-technical skills learning strategies for undergraduates: a systematic review. Ann Med Surg Lond. 2018;36:29-40.

19. Field MJ. Dental education at the crossroads: challenges and change. Inst Med Rep. 1995;Washington, DC: National Academy Press.

20. Formicola AJ. Trends in dental faculty of U.S. dental schools, 2003-04 to 201314. J Dent Educ. 2017;81(8 suppl):eS33-40.

21. Lantz MS, Shuler CF. Trends in basic sciences education in dental schools, 1999-2016. J Dent Educ. 2017:81(8) eS55-eS65.

22. Nicholson SL, Hayes MJ, Taylor JA. Cultural Competency Education in Academic Dental Institutions in Australia and New Zealand: A Survey Study. J Dent Educ. 2016;80(8):966-74

23. Wollersheim H, Hermens R, Hulscher M, et al. Clinical indicators: development and applications. Neth J Med. 2007;65(1):15-22.

24. Estay J, Martín J, Viera V, Valdivieso J, Bersezio C, Vildosola P, et al. 12 years of repair of amalgam and composite resins: a clinical study. Oper Dent. 2018;43(1):12 21.

25. Küster-Boluda I, Vila-López, N. El docente universitario y sus efectos en el estudiante. Estud Sobre Educ. 2012;23:157-82. 